\title{
DOS and Brute Force attacks faults detection using An Optimised Fuzzy C-means
}

\author{
Karwan Qader \\ School of Computing \\ University of Portsmouth \\ Portsmouth, UK \\ Email: karwan.qader@port.ac.uk
}

\author{
Mo Adda \\ School of Computing \\ University of Portsmouth \\ Portsmouth, UK \\ Email: mo.adda@port.ac.uk
}

\begin{abstract}
This paper explains how the commonly occurring DOS and Brute Force attacks on computer networks can be efficiently detected and network performance improved, which reduces costs and time. Therefore, network administrators attempt to instantly diagnose any network issues. The experimental work used the SNMP-MIB parameter datasets, which are collected via a specialised MIB dataset consisting of seven types of attack as noted in section three. To resolves such issues, this researched carried out several important contributions which are related to fault management concerns in computer network systems. A central task in the detection of the attacks relies on MIB feature behaviours using the suggested SFCM method. It was concluded that the DOS and Brute Force fault detection results for three different clustering methods demonstrated that the proposed SFCM detected every data point in the related group. Consequently, the FPC approached 1.0, its highest record, and an improved performance solution better than the EM methods and K-means are based on SNMP-MIB variables.
\end{abstract}

Index Terms-Fault detection, Fuzzy Cluster Means, Network Fault Attacks, Subtractive Clustering, Fault Clustering

\section{INTRODUCTION}

Recently, there has been an increase in the security threats which set challenges towards worldwide computer networks and IT economic progression. Network security and conditions are required factors for maintaining performance, systems must implement control networks regarding tolerance, adaptability, scalability, attainability, and for faults [1]. Regular network behaviour may be symbolised by different factors, e.g. the type of network data which is being measured, network traffic volume, and the kinds of applications which run on the network [2]. A network attack is errors which result from various factors, e.g. disruption to fibres, router failure, network attacks, etc. Additionally, each service disruption might not be the result of an error, and vice versa [3]. [4] suggests that network faults are usually the result of either a failure of devices or links, or traffic attacks, both of which can lead to traffic losses. Therefore, fault tolerance is a crucial network attribute [5]. It also signifies network capability in maintaining functionality irrespective of system errors. Both strong reliability and fault tolerance are coveted attributes of every network [6]. Attacks occurring in networks can result in packet loss and network faults which often occur in load balancers. Fault detection is a vital part of network performance maintenance in addition to the prevention of system breakdowns [4]. Detecting network anomalies is a main research focus and development in the intrusion detection field [7]. It may aid the network administration process and enable implementing appropriate actions which guarantee functionality of the network and service quality provision. A frequently used method of data mining in fault detection is clustering; this is used in applications which are used to detect network anomalies. The object groups which this procedure determines enable network administrators to make decisions which give protection to data network communications [8]. This study's objective is devising a process for detecting attacks and anomalies in the network through the use of datasets which incorporate the aspects of management information bases (MIB) variables in the local node. This is achieved by applying machine learning approaches which use Fuzzy Cluster Means (FCM) injected with Subtractive Clustering to overcome FCM limitations in identifying the initial cluster and cluster centres; these can accurately detect irregular traffic activity in the extant datasets. The primary contributions can be described as follows:

- Fuzzy Cluster Means (FCM) detects changes in the behaviour of Simple Network Management Protocol management information bases (SNMP-MIB) parameters. As far as we know, no other research applied FCM detects DOS and Brute force attacks in computer networks. FCM is rarely applied, but when it is the algorithm is in a different environment and the data is more diverse.

- To overcome the main FCM issues, known commonly for slow performance, we combined a new algorithm Subtractive Clustering (SC). The new FCM is suggested to be named as Subtractive FCM (SFCM).

- The optimised model very precisely detects anomalies because of its fuzziness characteristics and indicates each data point to be a member of a group of the attacks, where the majority of the clustering methods and other filtering miss the detection of some of the data points.

- The detection results are validated based on the Fuzzy Partition Coefficient to demonstrate the most efficient detection rate. The results show that the optimised Fuzzy model outperforms the K-means and Expectation Maximisation (EM). 
The remainder of the paper is organised as follows. Section 2 gives a brief review of related work in the same area. In section 3, the case study and dataset features, including all attack types, are presented. Section 4 shows the suggested clusterisation framework methods. Section 5 shows the results of the simulation with detailed analysis of the efficiency and performance. Lastly, a conclusion of the research work is provided in section 6 .

\section{RELATED WORK}

A cluster is an object group which has similarities and clustering is an established area in data mining. Many clustering definitions exist in the literature without a single precise definition [9]. A multitude of clustering techniques are consequently available. These are able to be divided into two different types, namely soft-clustering methods, grid-based clustering methods, model-based clustering methods, densitybased clustering methods, partition clustering methods, and hierarchical clustering methods. Every clustering method category uses a different approach dependent of their modus operandi and utility. Fuzzy cluster means (FCM) is a clustering technique which is derived from the K-Means algorithm. FCM and K-Means differ in that the latter refers to hard clustering whilst FCM is used for soft clustering. It produces soft clusters where objects might belong to more than one cluster with differing probabilities, making use of distance measure operating on objects situated in an n-dimensional vector [10]. This algorithm is broadly used in the data mining domain, particularly for pattern recognition. [11] originally developed the algorithm which was subsequently improved by [12].

[13] suggested a fault detection filter for improving fault identification quality. Both filtering and fault detection are achieved by addressing filtering and detection problems using a stochastic switched fuzzy time-delay system. [14] used a new algorithm based on the Adaptive Network-based Fuzzy Inference System (ANFIS) to classify transmission line faults. Faults were classified into Low Impedance Faults (LIF) and High Impedance Faults (HIF). [15] They suggested a hybrid algorithm for diagnosing faults based on fuzzy classification and artificial immune systems. This includes features such as classification, rule cleaning, and computation of support and confidence. Their method has become known as the Fuzzy Artificial Immune Recognition System (FAIRS). [16] suggested a method of fault detection based on Centre of Gravity (COG) defuzzification and Fuzzy Cluster Means (FCM). For signal separation, FCM is used whilst defuzzification is utilised for generating fused signals. [17] proposed, for fault detection and classification in two tank process industries, a hybrid mechanism Genetic Adaptive Neuro-Fuzzy Inference System (GANFIS). Techniques were employed such as Genetic Neuro-Fuzzy Systems and the Kalman Filter. [18] used fuzzy-ARTMAP in automotive application fault detection. It is a neural network using both conflict intersection and classification for classifying and isolating faults and enhancing performance.
[19] suggested a Fuzzy K-Nearest Neighbour (FK-NN) algorithm to locate and classify faults in transmission lines. They discovered that fuzzy K-NN demonstrated improved performance over the traditional K- NN. The article by [20] suggested an FCM technique in the form of an efficient fuzzy clustering-based algorithm to detect potential attacks in an efficient intrusion detection system for implementing mobile ad hoc networks (MANETs) into cloud-based storage environments. [21] discovered the availability and performance of cluster-based services. They used fault injection techniques and analytical modelling for evaluating the availability and performance of networks which use protocols, such as TCP and VIA.

[22] researched mobile networks for Key Performance Indicators (KPI) and alarms. They looked into the Long Time Their system which is comprised of two phases. In the first phase, Expectation Maximization (EM) was used for input data discretisation whilst in the second phase call status was identified. LTE network faults were then diagnosed. Fault detection is a vital network management task, as analysed by [23], for proposing a statistical method based on the Wiener filter for capturing abnormal behavioural changes of the MIB variables. The study algorithm took data from two different scenarios and four different case studies. A twophase approach to measure cluster based internet service performance was presented by [24]. The first methodology phase uses a fault-injection approach to measure the impacts of faults on network performance, whilst the second phase uses analytical models for assessing network performance by combining the first phase measurements and the fault loads. This two-phased approach allows an evaluator study into how the servers respond to different design-related decisions, fault rates, amongst other factors. Four PRESS web server versions were tested against five fault classes for measuring the server performance in different scenarios [25].

An ensemble Fault Diagnosis Based on Fuzzy C-means Algorithm of the Optimal Number of Clusters and Probabilistic Neural Network (FCM-ONC-PNN) was represented by [26] and showed how significant fault diagnosis is and which follows the maintaining of product quality in industrial systems.

Lastly, Fuzzy C-Means Clustering and a Feed Forward Neural Network were employed [27] to discover any faults in the software module, focusing on the early detection benefits of fault prone software components and how a hybrid approach based on fuzzy C-means clustering based approach and feedforward neural network based approach is used for finding faults.

\section{Network ATtACKS AND DATASETS}

The experimental datasets were extracted from research by [28] in a network topology, consisting of one PC router, 2 subnets, and 2 switches. The first subnet consisted of 5 PCs with one of them acting as the attacker. The second subnet consisted of 5 PCs with one acting as the server (victim). The researchers used the LanTrafficV2 in two phases. The 
first phase was for MIB data collection in a real environment, whilst the second phase tested the MIB data which was collected in attack detection. Several tools were used for conducting different configuration parameters, e.g. packet type (TCP, UDP, ICMP) to generate the number of packets sent, packet size, delay... etc. Normal traffic along with six types of DOS flooding attacks and Brute Force attack were conducted using the abovementioned tools with various parameters, as shown in table 1 .

\begin{tabular}{|l|l|}
\multicolumn{2}{|c|}{ TABLE 1. NETWORK ATTACKS AND tHE TOOLS USED } \\
\begin{tabular}{|c|l|l|}
\hline Attack type & Tool used \\
\hline Brute Force attack & THC-Hydra \\
\hline \multirow{4}{*}{$\begin{array}{c}\text { DOS } \\
\text { flooding } \\
\text { attacks }\end{array}$} & TCP-SYN flooding & HyenaeFE \\
\cline { 2 - 3 } & UDP flooding & HyenaeFE \\
\cline { 2 - 3 } & ICMP-ECHO flooding & HyenaeFE \\
\cline { 2 - 3 } & HTTP flood & DOSHTTP 2.5 .1 \\
\cline { 2 - 3 } & Slowloris & Slowloris script \\
\cline { 2 - 3 } & Slow post & HttpDosTool4.0 \\
\hline
\end{tabular}
\end{tabular}

They mainly used MIB variables, and from the 12 variable groups they selected the most appropriate network attack characteristics from the groups (Interface, IP, TCP, UDP and ICMP groups) in regard to the work environment as shown in table 2.

Nonetheless, in the process's final stages, the 34 MIB variables were collected every 15 seconds during the attack experiment, where the server (victim) was variously attacked. We collected 4998 MIB data records, including 600 normal MIB records, 960 TCP-SYN, 773 UDP flood, 632 ICMPECHO, 573 HTTP flood, 780 Slowloris, 480 Slowpost and 200 Brute Force attack records. A simple network management protocol (SNMP) gathered and arranged information regarding the instruments which pertained to an internet protocol (IP), including servers, printers, hubs, switches, and routers. An SNMP uses a management information base (MIB) to gather the information, incorporating multiple packets or octets which are distributed, delivered, received and discarded. The variables show higher sensitivity levels regarding traffic behaviour when attacks are injected.

\section{RESEARCH METHODS AND THE FRAMEWORK}

In computer traffic fault data, there exists uncertainty and inaccurate information emerging from the variables in relation to the Management Information Base (MIB) groups, defined as network entity groups which are controlled via a Simple Network Management Protocol (SNMP). Hard clustering, including k-means and Self Organizing Maps (SOM) are incompatible with data examination, since the traffic faults clusters intersect because of multiple MIB variables in every distinct flaw. Positive aspects exist of fuzzy theory regarding its approach to data management which contains some uncertainty. Fuzzy clustering methods are compatible with the fuzzy set theory, which accounts for uncertainty when examining the
TABLE 2. MIB PARAMETERS AND THEIR GROUPS

\begin{tabular}{|c|c|c|}
\hline Parameter No. & MIB Group & MIB Features \\
\hline P1 & \multirow{8}{*}{ Interface (IF) } & ifInOctets11 \\
\hline $\mathrm{P} 2$ & & ifOutOctets11 \\
\hline P3 & & ifoutDiscards11 \\
\hline P4 & & ifInUcastPkts11 \\
\hline P5 & & ifInNUcastPkts11 \\
\hline P6 & & ifInDiscards11 \\
\hline P7 & & ifOutUcastPkts11 \\
\hline P8 & & ifOutNUcastPkts11 \\
\hline P9 & \multirow{8}{*}{ TCP } & tcpOutRsts \\
\hline $\mathrm{P} 10$ & & tcpInSegs \\
\hline P11 & & tcpOutSegs \\
\hline $\mathrm{P} 12$ & & tcpPassiveOpens \\
\hline P13 & & tcpRetransSegs \\
\hline P14 & & tcpCurrEstab \\
\hline $\mathrm{P} 15$ & & tcpEstabResets \\
\hline P16 & & tcpActiveOpens \\
\hline P17 & \multirow{4}{*}{ UDP } & udpInDatagrams \\
\hline P18 & & udpOutDatagrams \\
\hline P19 & & udpInErrors \\
\hline $\mathrm{P} 20$ & & udpNoPorts \\
\hline $\mathrm{P} 21$ & \multirow{8}{*}{ IP } & ipInReceives \\
\hline $\mathrm{P} 22$ & & ipInDelivers \\
\hline $\mathrm{P} 23$ & & ipOutRequests \\
\hline $\mathrm{P} 24$ & & ipOutDiscards \\
\hline $\mathrm{P} 25$ & & ipInDiscards \\
\hline $\mathrm{P} 26$ & & ipForwDatagrams \\
\hline $\mathrm{P} 27$ & & ipOutNoRoutes \\
\hline $\mathrm{P} 28$ & & ipInAddrErrors \\
\hline P29 & \multirow{6}{*}{ ICMP } & icmpInMsgs \\
\hline $\mathrm{P} 30$ & & icmpInDestUnreachs \\
\hline P31 & & icmpOutMsgs \\
\hline P32 & & icmpOutDestUnreachs \\
\hline P33 & & icmpInEchos \\
\hline P34 & & icmpOutEchoReps \\
\hline
\end{tabular}

data features of the Simple Network Management Protocol (SNMP). Therefore, all feature vectors in the dataset are assigned a membership value with each group, implying how much it belongs to the cluster bezdek1981objective. Fuzzy clustering aims to provide cluster definitions by precisely determining its membership function, which is beneficial to resolve the high dimensionality issue and for detecting any potential network attacks. It is seen as an iteratively optimal algorithm with a greater degree of flexibility, especially for traffic examination.

\section{A. Subtractive fuzzy cluster means (SFCM)}

A widely used clustering method is Fuzzy c-means, since its performance exceeds the clustering traffic fault data process. Nonetheless, this algorithm has drawbacks in ascertaining the number of clusters; at the start the clusters can be quite lengthy. Additionally, an excessive iterations are needed to optimise the initial and optimal cluster numbers. Because FCM performance is reduced when attempting to resolve these issues, an additional algorithm defined as subtractive clustering (SC) is introduced to the FCM to resolve this problem. The main SFCM analysis steps shown in the (Proposed algorithm Optimised FCM) are:

- Identify the original data clusters 
- Provide a definition of subtractive clustering

- Determine the number of original clusters based Subtractive data

- Validate the original data clusters.

Cluster analysis has great strength in data analysis and has been applied successfully to the researches of various fields. an excessive amount of iterations are required in the optimisation of the initial and optimal cluster numbers. As the performance of FCM is reduced in the process of attempting to resolve these problems, an additional algorithm defined as subtractive clustering (SC) is introduced into the FCM in order to resolve the aforementioned problem. The primary objective of utilising FCM is its contribution to both grouping and segmentation. Eventually the new developed algorithm is proposed to detect any anomalies when attacks occur in the network system as depicted in the below:

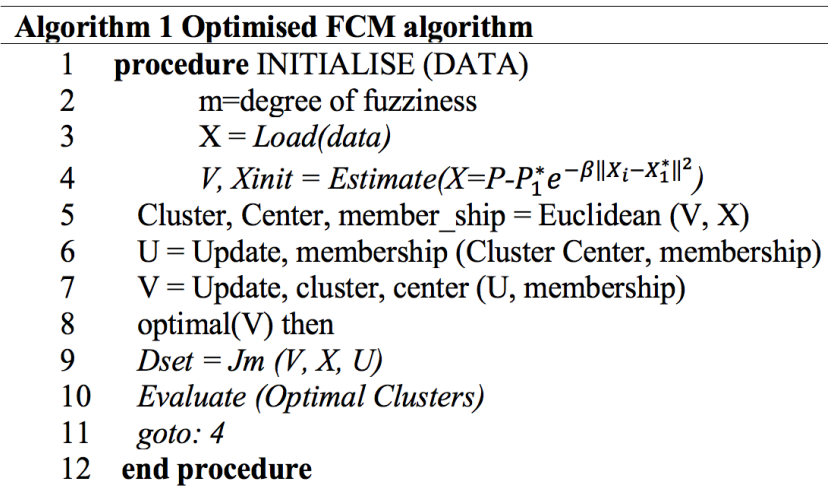

\section{B. Proposed Frame Work}

A widely used clustering method is Fuzzy c-means, since its performance exceeds the clustering traffic fault data process. Nonetheless, there are drawbacks to this algorithm in ascertaining the number of clusters, and the clusters at the beginning can be quite long. Additionally, excessive iterations are needed to optimise the initial and optimal cluster numbers. When FCM performance is reduced when trying to resolve these issues, another algorithm defined as subtractive clustering (SC) is used in the FCM to resolve the problem, as shown in figure (The proposed framework of the SFCM). The main aim of using FCM is its contribution to detecting all occurring attacks and grouping them. Yet, when there are different attacks causing simultaneous multiple faults this may lead to network traffic because of the magnitude. To prevent this problem, unsupervised machine learning is used to cluster the different datasets originating from diverse segments in the network traffic. Subsequently, each occurring cluster is optimised and the output feature vectors are moved to the subsequent stages so that every system fault can be detected appropriately. FCM is composed of different mathematical equations used to minimise the objective functions, this may be achieved via optimising the degree of membership and cluster centres at each of the iterations as shown in figure 1 .

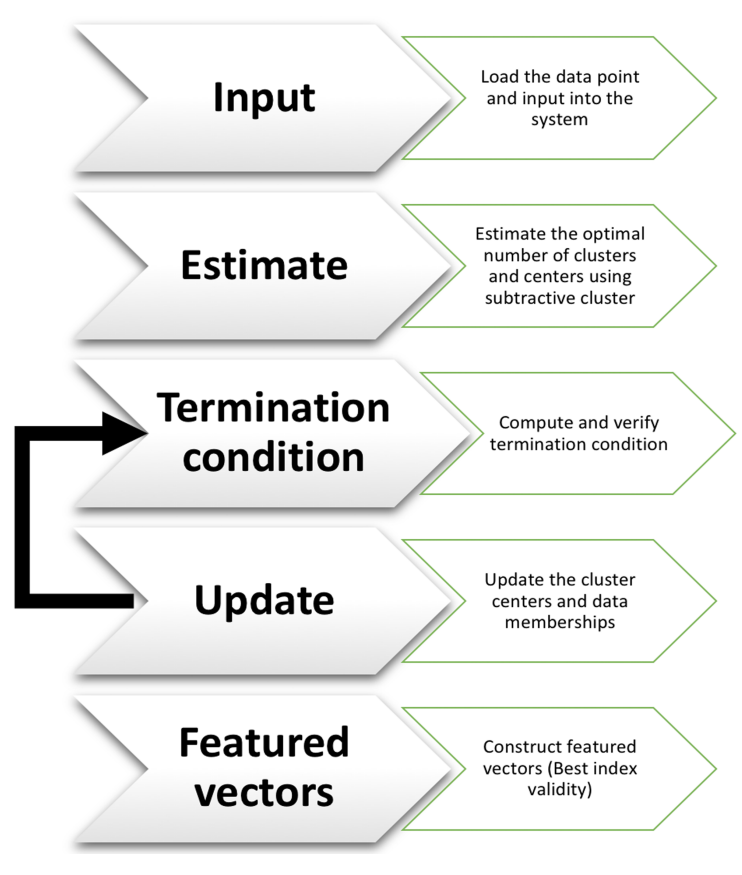

Fig. 1. The proposed SFCM framework

Additionally, the FCM technique is useful for managing vague cluster boundaries. It is essential that the high dimensionality issued is resolved. FCM is an algorithm which is iteratively optimised and which has greater flexibility, particularly for traffic analysis.

\section{Network Attacks Detection Based on Clustering Methods}

Using the unsupervised method can precisely detect any network attacks by segmenting each fault pattern and form groups for detected traffic. Therefore, this process enriches the research to provide an application for detecting anomalies in real time and overwhelm Big Data, since concern in the research seeks to find a suitable solution for resolve the issues presented by Big Data.

\section{A. Clustering methods performances}

In line with previous work, Fuzzy C-means, K-means and Expectation Maximisation (EM) are three common cluster algorithms and are chosen and applied to dataset samples. We realise that, because of the nature of its fuzziness, FCM achieved optimal clustering results where every data point can be a member of whichever cluster group.

We solved the performance issue as the main FCM limitation and improved the performance results by overcoming the initial cluster centres by consolidating a subtractive clustering method with the FCM. We produced data from 64,000 data points and used different tests to compare the performance of each method. Figure 2 shows three different cluster algorithm performances (Subtractive Fuzzy Clustering Means [SFCM], $\mathrm{K}$-means and EM) used in the experiment.

As shown on the graph, the optimised model (SFCM) competed with the K-means with approximately the same 


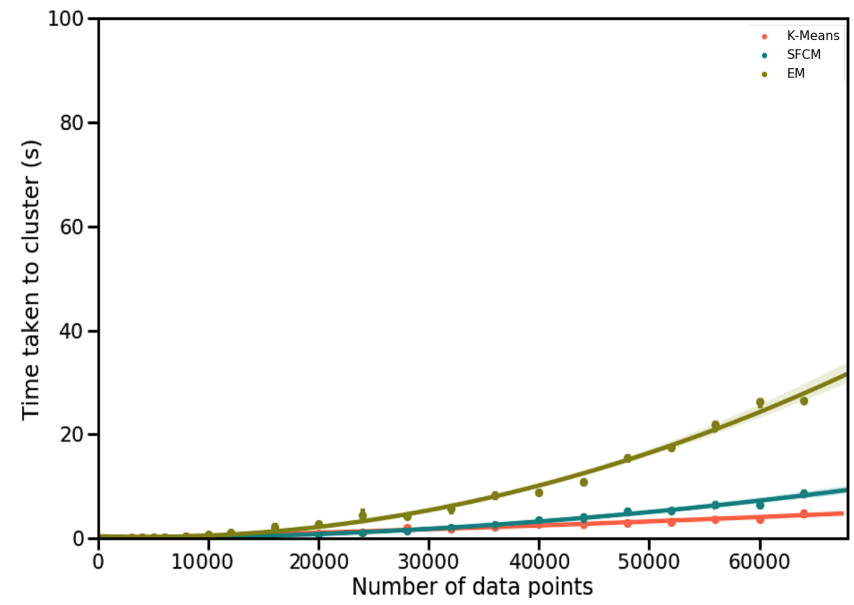

Fig. 2. Performance comparison of K-means, SFCM and EM implementations

performance over the clustering of 45,000 data points, whilst the $\mathrm{K}$-means has the best performance. After the data is scaled out, the SFCM was slower than the K-means, when the time taken increased with the total of the data points implemented, which was around seven seconds. Furthermore, the result demonstrated that SFCM achieved an improved performance over EM, which required 32 seconds to complete the implementation.

\section{B. Experiment Results Discussion and Evaluations}

Therefore, amongst the existing clustering methods, the hybrid SFCM, K-means and EM are utilised to apply to the datasets for the optimal number of clusters. Clustering is crucial in the fault diagnosis process, through detecting all data points of the attacks accurately in its group.

Our samples indicate that the dataset includes all seven different DOS attacks and Brute Force attacks using several libraries in Python, such as Numpy, Pandas and Scikit. For data analysis we clustered our dataset, it needs to reach its optimum with 8 clusters, multiple times, with between two and nine clusters, as shown in Figure 3.

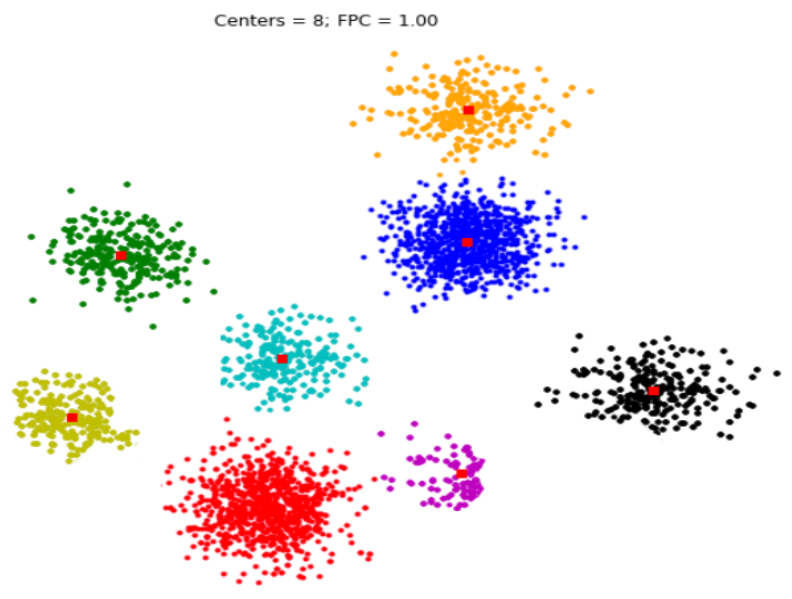

Fig. 3. The PC of the proposed SFCM for eight centres
The partition coefficient (PC) is a measurement metric which defines the range 0 to 1 , with one being the best. It is a metric indicating purity where a specific model describes our data and is calculated using the following equation:

$$
V_{p c}=\frac{1}{N} \sum_{i=1}^{c} \sum_{j=1}^{N}\left(\mu_{i j}\right)^{2}
$$

$\mathrm{N}$ represents the number of data points, $\mathrm{c}$ is the cluster numbers and uij pictures the membership function degree vector.

The results in Figure 3 show that SFCM PC is the greatest, with points accurately detected and approaching 1.0 when gathering eight different clusters; it can therefore be stated that SFCM reached the highest optimal cluster value. For the same cluster numbers using K-means and EM methods, as shown in Figures 4 and 5, the PC was minimised and achieved 0.62 and 0.71 , respectively.

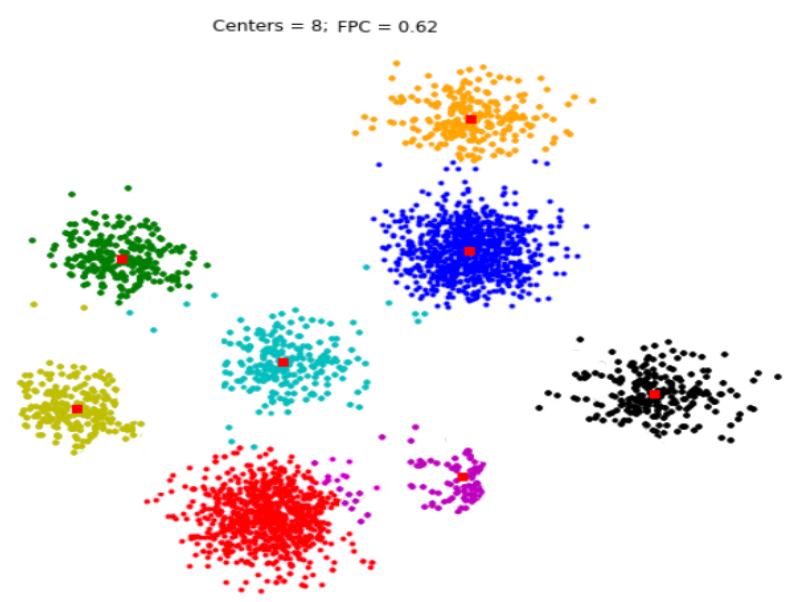

Fig. 4. The PC of the proposed K-means for eight centres

Here, applying K-means and EM proves that these two methods are unsuitable for this type of data to achieve wellcompacted clusters.

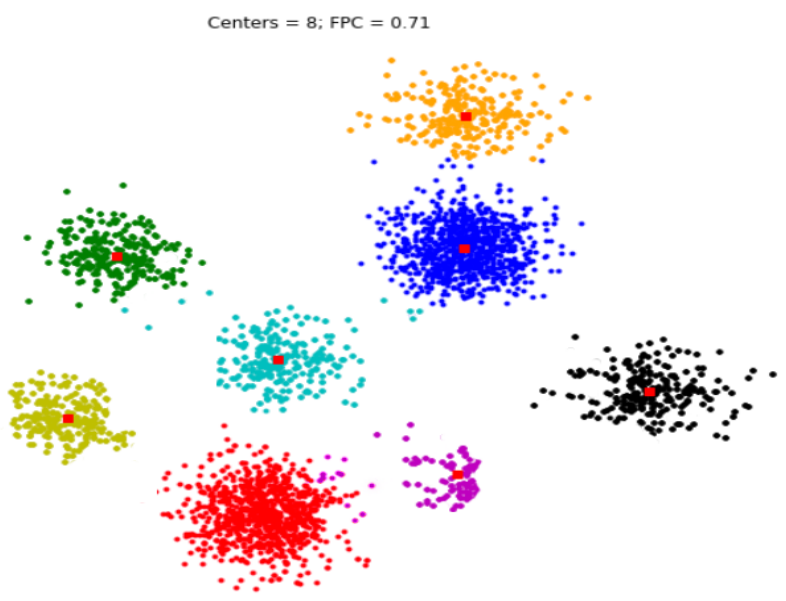

Fig. 5. The PC of the proposed EM for eight centres 
Each cluster in Figure 3, 4 and 5 represents a specific attack with its abnormal traffic. We demonstrate the clustering results and plot the partition coefficient (PC) for every method. When the PC is maximised, our data is best described and in this sample the optimal cluster number achieves its peak when it has eight clusters based on SFCM and, conversely, has a poor outcome when K-means and EM are applied, as shown in Figure 3.

\section{CONCLusion}

This study contributes to many of the significant novelties in the detection of DOS attacks and Brute Force attacks in computer network systems using SFCM. The FCM is used to breakdown the complex data into segments for the accurate detection of occurrence attacks in real-time. The experiment proves that the FCM (Fuzzy Cluster) reached more efficient cluster results than K-means (Hard Cluster) and EM (Hard and soft Clusters). We made improvements to the FCM algorithm and developed Subtractive FCM (SFCM) through the combination of a Subtractive Cluster for performance improvement to the FCM algorithm, this was practically approved as shown in figure 2. To resolve the FCM constraints regarding determining the number of starting clusters and the cluster centres, a subtractive clustering algorithm was used in the FCM, directly augmenting and optimising the cluster outcomes.

In total, it validate that the proposed SFCM outperforms the $\mathrm{K}$-means and EM where the partition coefficient (PC) reached (1.0).

\section{REFERENCES}

[1] K. Qader, M. Adda, and M. Al-Kasassbeh, "Comparative analysis of clustering techniques in network traffic faults classification," International Journal of Innovative Research in Computer and Communication Engineering, vol. 5, no. 4, pp. 6551-6563, 2017.

[2] D. B. Roy and R. Chaki, "State of the art analysis of network traffic anomaly detection," in 2014 Applications and Innovations in Mobile Computing (AIMoC). IEEE, 2014, pp. 186-192.

[3] K.-J. Cho, S.-J. Ahn, and J.-W. Chung, "A study on the classified model and the agent collaboration model for network configuration fault management," Knowledge-Based Systems, vol. 16, no. 4, pp. 177-190, 2003.

[4] P. Gill, N. Jain, and N. Nagappan, "Understanding network failures in data centers: measurement, analysis, and implications," in ACM SIGCOMM Computer Communication Review, vol. 41, no. 4. ACM, 2011, pp. 350-361.

[5] A. Angjeli, E. Cheng, and L. Lipták, "Linearly many faults in dualcube-like networks," Theoretical Computer Science, vol. 472, pp. 1-8, 2013.

[6] F. Bistouni and M. Jahanshahi, "Pars network: a multistage interconnection network with fault-tolerance capability," Journal of Parallel and Distributed Computing, vol. 75, pp. 168-183, 2015.

[7] M. H. Bhuyan, D. K. Bhattacharyya, and J. K. Kalita, "Network anomaly detection: methods, systems and tools," Ieee communications surveys \& tutorials, vol. 16, no. 1, pp. 303-336, 2014.

[8] K. Qader and M. Adda, "Fault classification system for computer networks using fuzzy probabilistic neural network classifier (fpnnc)," in International Conference on Engineering Applications of Neural Networks. Springer, 2014, pp. 217-226.

[9] T. De, D. Fraix Burnet, and A. K. Chattopadhyay, "Clustering large number of extragalactic spectra of galaxies and quasars through canopies," Communications in Statistics-Theory and Methods, vol. 45, no. 9, pp. 2638-2653, 2016.

[10] M.-S. Yang, "A survey of fuzzy clustering," Mathematical and Computer modelling, vol. 18, no. 11, pp. 1-16, 1993.
[11] J. C. Dunn, "A fuzzy relative of the isodata process and its use in detecting compact well-separated clusters," 1973.

[12] J. C. Bezdek, R. Ehrlich, and W. Full, "Fcm: The fuzzy c-means clustering algorithm,' Computers \& Geosciences, vol. 10, no. 2-3, pp. 191-203, 1984

[13] D. Zhang, L. Yu, and Q.-G. Wang, "Fault detection for networkbased nonlinear systems with communication constraints and missing measurements," in Control Conference (ASCC), 2011 8th Asian. IEEE, 2011, pp. 181-186.

[14] S.-M. Yeo, C.-H. Kim, K. Hong, Y. Lim, R. Aggarwal, A. Johns, and M. Choi, "A novel algorithm for fault classification in transmission lines using a combined adaptive network and fuzzy inference system," International journal of electrical power \& energy systems, vol. 25, no. 9, pp. 747-758, 2003.

[15] L. Xu and M.-Y. Chow, "Power distribution system fault diagnosis using hybrid algorithm of fuzzy classification and artificial immune systems," in Soft Computing Applications in Industry. Springer, 2008, pp. 357372.

[16] E. ElMadbouly, A. Abdalla, and G. M. ElBanby, "Sensor fusion and sensor fault detection with fuzzy clustering," in Computer Engineering and Systems (ICCES), 2010 International Conference on. IEEE, 2010, pp. 265-268.

[17] H. M. Khalid, A. Khoukhi, and F. M. Al-Sunni, "Fault detection and classification using kalman filter and genetic neuro-fuzzy systems," in Proceedings of the Annual Meeting of the North American Fuzzy Information Processing Society, El Paso, TX, USA, 2011, pp. 18-20.

[18] M. Benkaci, A. Doncescu, and B. Jammes, "Fault detection and isolation using fuzzy-artmap classification and conflict intersection," in Broadband and Wireless Computing, Communication and Applications (BWCCA), 2011 International Conference on. IEEE, 2011, pp. 441446.

[19] Y. Zhang, J. Chen, Q. Fang, and Z. Ye, "Fault analysis and prediction of transmission line based on fuzzy k-nearest neighbor algorithm." in ICNC-FSKD, 2016, pp. 894-899.

[20] M. Chen, N. Wang, H. Zhou, and Y. Chen, "Fcm technique for efficient intrusion detection system for wireless networks in cloud environment," Computers \& Electrical Engineering, 2017.

[21] K. Nagaraja, N. Krishnan, R. Bianchini, R. P. Martin, and T. D. Nguyen, "Evaluating the impact of communication architecture on the performability of cluster-based services," in High-Performance Computer Architecture, 2003. HPCA-9 2003. Proceedings. The Ninth International Symposium on. IEEE, 2003, pp. 229-240.

[22] L. Flores-Martos, A. Gomez-Andrades, R. Barco, and I. Serrano, "Unsupervised system for diagnosis in lte networks using bayesian networks," in Vehicular Technology Conference (VTC Spring), 2015 IEEE 81st. IEEE, 2015, pp. 1-5.

[23] M. Al-Kasassbeh and M. Adda, "Analysis of mobile agents in network fault management," Journal of Network and Computer Applications, vol. 31, no. 4, pp. 699-711, 2008.

[24] Y.-H. Song, A. Johns, Q. Xuan, and J. Liu, "Genetic algorithm based neural networks applied to fault classification for ehv transmission lines with a upfc," 1997.

[25] B. Zhang, Z. He, and Q. Qian, "Application of wavelet entropy and adaptive nerve-fuzzy inference to fault classification," in 2006 International Conference on Power System Technology. IEEE, 2006, pp. 1-6.

[26] Q. Yang, J. Guo, D. Zhang, and C. Liu, "Fault diagnosis based on fuzzy c-means algorithm of the optimal number of clusters and probabilistic neural network," International Journal of Intelligent Engineering \& Systems, vol. 4, no. 2, pp. 51-59, 2011.

[27] K. Purswani, P. Dalal, A. Panwar, and K. Dashora, "Software fault prediction using fuzzy c-means clustering and feed forward neural network," International Journal of Digital Application \& Contemporary Research, vol. 2, no. 1, 2013.

[28] G. Al-Naymat, M. Al-Kasassbeh, and E. Al-Harwari, "Using machine learning methods for detecting network anomalies within snmp-mib dataset," International Journal of Wireless and Mobile Computing, vol. 15, no. 1, pp. 67-76, 2018. 